\title{
MOOD ASPECTS OF RAPID WEIGHT LOSS IN ADOLESCENT WRESTLERS
}

\author{
Hrvoje Karninčić1, Mario Baić ${ }^{2}$, and Kristijan Slačanac ${ }^{2}$ \\ ${ }^{1}$ Faculty of Kinesiology, University of Split, Croatia \\ ${ }^{2}$ Faculty of Kinesiology, University of Zagreb, Croatia
}

Original scientific paper

UDC: 613.2:796.81-053.6

\begin{abstract}
:
The aims of this paper were: a) to present weight reduction habits of adolescent wrestlers; b) to establish differences in mood states between weight groups; and c) to determine relations between weight loss and indicators of hydration with mood states. Adolescent wrestlers do resort to rapid weight loss (RWL) regimens in their training process and competition $-70.1 \%$ of the subjects reduced their body mass and the reduction regime usually lasted one week. Similar habits have been reported in other studies despite the well evidenced detrimental effects of RWL. Urine specific gravity (USG) values suggest that all the subjects were in the state of dehydration ranging from a mild to a high one. The groups of low weight (LW) and middle weight (MW) wrestlers reduced their body mass significantly more than others $(B M ; p<.05)$ and their sensation of fatigue was significantly higher $(\mathrm{p}<.05)$. The variables body mass reduction and \%BM reduction were correlated with negative mood states, whereas the indicators of hydration in one of the groups (MW) established ambiguous relations with dehydration and positive mood states.
\end{abstract}

Key words: Brunel's mood scale, dehydration, combat sport, specific urine gravity

\section{Introduction}

In wrestling there is a trend of rapid weight loss (RWL), like in most other combat sports. The target of RWL appears to be a contest in a lower weight category because wrestlers believe they gain a series of advantages over the opponents in that way. Namely, wrestlers of a higher body mass (BM) are stronger than lighter athletes (Song \& Garvie, 1980; Yoon, 2002). The wrestlers who reduce their body mass rapidly regain their mass between the weigh-in and contest thus becoming heavier than their competition opponents (Horswill, Scott, Dick, \& Hayes, 1994; Utter, 2001). Although there are studies that associate RWL and good performance in wrestling, they are scarce and inconclusive (Franchini, Brito, \& Artioli, 2012). Even more, the ways in which wrestlers reduce their BM have, as a rule, a detrimental effect on their health (Artioli, Franchini, et al., 2010). Harmful effects regard mostly the methods of a short-term body mass reduction, like extreme dehydration, impermeable (sweat-inducing) suits wearing, as well as usage of diuretics, laxatives, provoked vomiting, and extremely strenuous exercising in very warm environments (Remick, et al., 1998; Weber, et al., 2013). Several deaths, caused by the applied methods, suggest that RWL is a serious problem (Remick, et al., 1998).
As for the age of cadet wrestlers (16-17 years of age), they are in midst of their intensive physical growth and development. Previous research has demonstrated that cadet wrestlers are well acquainted with RWL (Franchini, et al., 2012; Kordi, Ziaee, Rostami, \& Wallace, 2011; Tipton \& Tcheng, 1970) despite the fact that RWL has been evidenced as a risk factor of disorders in somatic development (McMurray, Proctor, \& Wilson, 1991; Smith, 1984) as well as of eating disorders (Oppliger, Landry, Foster, \& Lambrecht, 1993; Saarni, Rissanen, Sarna, Koskenvuo, \& Kaprio, 2006; Steen \& Brownell, 1990). Eating disorders are not exclusive psychological disorders associated with RWL. Acute dehydration states and RWL have an impact on cognitive functioning (Choma, Sforzo, $\&$ Keller, 1998). Research studies on psychological effects of RWL have reported loss of concentration, self-confidence, short-term memory, as well as increased anger, confusion, depression and fatigue (Degoutte, et al., 2006; Horswill, Park, \& Roemmich, 1990; Steen \& Brownell, 1990). Concussion sustained in a wrestling combat will cause numerous and more intensive mental complications when combined with acute dehydration (Weber, et al., 2013).

All research studies but one have indicated negative psychological aspects of RWL; the excep- 
tion is a study presenting a theory that wrestlers successful in RWL gain mental advantage over their opponents (Pettersson, Ekstrom, \& Berg, 2013). The theory has it that only those wrestlers who do not give up and are prepared to do great sacrifices can significantly reduce their body mass. Wrestlers have not changed their habits of body mass reduction for decades although scientific evidence of harmful effects of RWL appeared already in the 1970s and 1980s (Klinzing \& Karpowicz, 1986; Tipton \& Tcheng, 1970). Research studies on associations between RWL and wrestling performance have not been conclusive; however, they managed to relate RWL closely to various harmful side effects. Our study focused on two crucial issues: rapid weight loss habits of wrestlers who were in a very sensitive stage of their growth and maturation, and their postRWL mood states. Therefore, a general objective was to establish relations between RWL and mood states in wrestling. In accordance with the preset objective, we hypothesized that there were significant differences between wrestlers (pertaining to different weight categories) in hydration status (after body mass reduction), and that there were significant differences between wrestlers (pertaining to different weight categories) in mood states after body mass reduction.

\section{Methods and measures}

The sample of subjects consisted of 77 male cadet wrestlers ( $16.0 \pm 0.8$ years of age). All the wrestlers participated in the Croatian National GraecoRoman Wrestling Championship (the total number of contestants was 87). The sample of subjects was representative for the population of Croatian cadet wrestlers since it encompassed $88.5 \%$ of all Croatian cadet wrestlers. The subjects were divided into three weight groups according to previous research on combat sports (Karnincic, Krstulovic, \& Baic, 2013; Khanna \& Manna, 2006): lightweight group (LW) comprising the official weight categories of 42, 46, 52 and $54 \mathrm{~kg}$; middleweight group (MW) with the official categories of 58, 63 and $69 \mathrm{~kg}$; and heavyweight group (HW) with the official categories of 76,85 and $100 \mathrm{~kg}$. The subjects' characteristics are presented to detail in Table 2.

Participation in this study was voluntary and anonymous for the cadet wrestlers who had to produce written parental permission. Research ethics review was carried out by the Research Ethics Committee of the Faculty of Kinesiology, University of Zagreb.

The sample of variables consisted of a 13-variable battery that can be divided into three groups. The first group of variables embraced eight psychological variables assessing mood states by means of Brunel's mood scale (BRUMS): anger, tension, depression, vigour, fatigue, confusion, happiness, and calmness. The second group of variables contained factors associated with body mass reduction: self-reported amount of the reduced body mass (weight loss in $\mathrm{kg}$ ), and percentage of the reduced body mass obtained in relation to the total body mass (rate of reduction). The third group of variables consisted of three non-invasive field tests objectively assessing hydration status: urine specific gravity (USG), urine colour and urine stripe test.

Brunel's mood scale is a reliable assessment tool (Lan, Lane, Roy, \& Hanin, 2012) with satisfactory psychometric characteristics suitable for the sample of adolescents (Zhang, Si, Chung, Du, \& Terry, 2014); it has already been used in research on combat sports (Stevens, Lane, \& Terry, 2006) and specifically on wrestling (Marttinen, Judelson, Wiersma, \& Coburn, 2011). It was introduced to the subjects in the following way: "Please, read each statement carefully and then circle the answer that best describes how you feel right now. Make sure you respond to every word". BRUMS is a psychological tool that measures current mood states using 24 mood descriptors of anger, confusion, depression, fatigue, tension, happiness, and vigour. Degrees in the BRUMS are specified as follows: 0 - not at all, 1 - a little, 2 - moderately, 3 - quite a bit, and 4 - extremely.

Hydration status of the subjects was assessed using three non-invasive tests already validated on the sample of wrestlers (Abbey, Heelan, Brown, \& Bartee, 2014; Fernandez-Elias, et al., 2014; Oppliger, Magnes, Popowski, \& Gisolfi, 2005): two tests measuring specific urine gravity, and one test assessing hydration via urine colour.

Refractometry measures specific urine gravity (USG). It is defined as the weight of urine volume compared to the same volume of distilled water. A refractometer AtagoPal 10s Tokyo, Japan, was used for USG measurements. Specific urine weight ranged from 1.000 to 1.040 with the deviation on a 0.001 scale. After the distilled water calibration, urine specimens were loaded in the device and the analysis results were read on the display.

Urine strips test measures specific urine weight by means of urine reagent strips. The test is based on changes of polyelectrolytes in the solution with the transformed ionic environment. Strips record changes of 0.005 in the range between 1.000 and 1.035. A reagent strips is dipped into a urine sample. After a short time it is taken out of the container and excessive urine is removed. After 45 seconds the dipped end is compared to the values printed on the strips' container. We used COMBINA 10 strips (HUMAN Gesellschaft für Biochemica und Diagnostica $\mathrm{mbH}$, Wiesbaden, Germany).

Urine colour test was used to assess hydration status through urine colour. Urobilin or urochrome is a pigment in urine. When a large amount of urine is excreted (high hydration), urine is transparent and pale. When a small amount of urine is excreted (dehydration), it is concentrated and dark 
(Shirreffs, 2003). Armstrong has designed and described the urine colour test assessing hydration status (Armstrong, 2005, 2007). The scale from 1 to 8 consists of colour models, from pale yellow (1) to dark brown (8). A urine sample, in a transparent container, is compared to the scaled colour spectrum in a well-lit room to find a corresponding colour.

All the subjects arrived to the sports hall at 8 a.m., and received their urine containers and an anamnestic questionnaire about their current BM reduction as well as about their habits connected therewith. They also received detailed instructions regarding the research procedure. The official weigh-in was scheduled for 9 a.m. and up to that time the subjects handed out their urine specimens and filled-in questionnaires. Urine specimens were immediately analysed for urine specific gravity (USG) and urine colour by the same experienced investigator. After the weigh-in, but prior to the contest, the subjects filled in the psychological questionnaire for the assessment of mood states.

All the data were analysed by using the Statistics 11 software package (Statsoft, USA). Descriptive statistics (mean $\pm \mathrm{SD}$ ) of all variables was calculated. Kolmogorov-Smirnov test was used to verify the data goodness of fit as regards the normality of distribution. Since the data did not meet parametric statistical criteria, they were further processed by non-parametric statistical methods. Differences between the groups were analysed by Mann-Whitney
U-test, whereas relations between mood states and objective indicators of hydration levels were determined by the Spearman's rank correlation coefficient.

\section{Results}

Out of 77 subjects, 54 (70.1\%) reduced their body mass. If the total subject sample is regarded, the wrestlers reduced their body mass by $2.00 \mathrm{~kg}$ on average, which is $1.25 \%$ of their total body mass. No subject reduced his body mass during one month period, only six subjects made their reduced weight over the period of two to three weeks, whereas even 48 subjects reduced their BM in the last pre-competition week.

As it is obvious from Table 1, all the three hydration tests were statistically significantly correlated at the level of the whole subject sample. However, from the aspect of subsamples, the USG (strips) test was not significantly correlated with the USG (refractometer) test. The correlation between the USG (refractometer) and urine colour tests was high and significant only for the LW and MW groups. Since no consistent and high enough correlation was established among the USG (strips), urine colour and USG (refractometer) tests, the last being the golden standard method in the hydration state non-invasive assessment, only the USG (refractometer) test results were used in further analysis.

Table 2 presents descriptive statistical parameters of participants' characteristics and factors

Table 1. Correlations (Spearman's rank correlation coefficient) among three non-invasive field tests assessing hydration status

\begin{tabular}{lcccccccc}
\hline & \multicolumn{2}{c}{ All groups $(\mathrm{N}=77)$} & \multicolumn{2}{c}{ LW $(\mathrm{n}=30)$} & \multicolumn{2}{c}{ MW $(\mathrm{n}=29)$} & \multicolumn{2}{c}{ HW $(\mathrm{n}=18)$} \\
\cline { 2 - 9 } & USG ref. & Colour & USG ref. & Colour & USG ref. & Colour & USG ref. & Colour \\
\hline USG Strips & $.29^{*}$ & $.31^{*}$ & .22 & .22 & .35 & .29 & .24 & $.49^{*}$ \\
USG ref. & & $.78^{*}$ & & $.92^{*}$ & & $.82^{*}$ & & .45 \\
\hline
\end{tabular}

* Correlations are significant at $p<.05$

Table 2. Descriptive statistical parameters - arithmetic means, standard deviations and differences (Mann-Whitney U-test) among the groups for the subjects' characteristics and factors associated with body mass reduction

\begin{tabular}{lcccccc}
\hline & \multicolumn{2}{c}{ Lightweight $(\mathrm{n}=30)$} & \multicolumn{2}{c}{ Middleweight $(\mathrm{n}=29)$} & \multicolumn{2}{c}{ Heavyweight $(\mathrm{n}=18)$} \\
\cline { 2 - 7 } Variables & $\chi \pm \mathrm{SD}$ & $\mathrm{LW}$ vs MW & $\chi \pm S \mathrm{D}$ & $\mathrm{MW}$ vs HW & $\chi \pm S D$ & LW vs HW \\
\hline Age (years) & $15.8 \pm 0.9$ & $\mathrm{p}=.12$ & $16.2 \pm 0.8$ & $\mathrm{p}=.69$ & $16.1 \pm 0.8$ & $\mathrm{p}=.29$ \\
Body mass $(\mathrm{kg})$ & $55.2 \pm 13.6$ & $\mathrm{p}<.001$ & $65.5 \pm 2.8$ & $\mathrm{p}<.001$ & $81.8 \pm 8.7$ & $\mathrm{p}<.001$ \\
Body height $(\mathrm{cm})$ & $167.8 \pm 7.2$ & $\mathrm{p}<.001$ & $176.3 \pm 6.8$ & $\mathrm{p}=.07$ & $179.2 \pm 3.5$ & $\mathrm{p}<.001$ \\
BMI & $18.3 \pm 2.3$ & $\mathrm{p}<.001$ & $21.1 \pm 1.6$ & $\mathrm{p}<.001$ & $25.5 \pm 2.5$ & $\mathrm{p}<.001$ \\
Experience (years) & $5.4 \pm 2.3$ & $\mathrm{p}=.74$ & $5.1 \pm 2.8$ & $\mathrm{p}=.69$ & $4.7 \pm 2.1$ & $\mathrm{p}=.37$ \\
Ranking & $5.3 \pm 3.2$ & $\mathrm{p}=.04$ & $7.7 \pm 4.5$ & $\mathrm{p}<.001$ & $3.6 \pm 2.1$ & $\mathrm{p}=.07$ \\
\hline \% BM reduction & $1.2 \pm 0.9$ & $\mathrm{p}=.59$ & $1.5 \pm 1.3$ & $\mathrm{p}=.09$ & $1.0 \pm 1.6$ & $\mathrm{p}=.08$ \\
BM reduction & $2.2 \pm 1.7$ & $\mathrm{p}=.78$ & $2.3 \pm 2.0$ & $\mathrm{p}=.04$ & $1.2 \pm 1.8$ & $\mathrm{p}=.02$ \\
USG strips & $1.028 \pm 0.005$ & $\mathrm{p}=.81$ & $1.028 \pm 0.005$ & $\mathrm{p}=.91$ & $1.028 \pm 0.005$ & $\mathrm{p}=.62$ \\
USG refrac. & $1.026 \pm 0.006$ & $\mathrm{p}=.81$ & $1.026 \pm 0.006$ & $\mathrm{p}=.96$ & $1.027 \pm 0.004$ & $\mathrm{p}=.95$ \\
Urine colour & $5.0 \pm 2.1$ & $\mathrm{p}=.99$ & $4.9 \pm 2.3$ & $\mathrm{p}=.61$ & $5.3 \pm 2.0$ & $\mathrm{p}=.61$ \\
\hline
\end{tabular}

Note. \% BM reduction - reduction in \% of body mass; BMI - body mass index; LW - lightweight category; MW - middleweight category; $\mathrm{HW}$ - heavyweight category; $\chi$ - arithmetic mean; SD - standard deviation; $\mathrm{p}$ - statistically significant difference. 
Table 3. Reliability of the Brunel Mood Scale (BRUMS) for all groups

\begin{tabular}{lccc}
\hline & Lightweight & Middleweight & Heavyweight \\
\hline Cronbach's alpha & .89 & .88 & .81 \\
Average inter-item correlation & .24 & .21 & .14 \\
\hline
\end{tabular}

Table 4. Differences between the groups (Mann-Whitney U-test) in Brunel Mood Scale (BRUMS)

\begin{tabular}{lcccccc}
\hline & LW & LW vs MW & MW & MW vs HW & HW & \multicolumn{2}{c}{ LW vs HW } \\
\cline { 2 - 7 } & $\chi \pm S D$ & $\mathrm{p}$ & $\chi \pm \mathrm{SD}$ & $\mathrm{p}$ & $\chi \pm S D$ & $\mathrm{p}$ \\
\hline Anger & $3.4 \pm 4.1$ & .68 & $2.9 \pm 3.3$ & .44 & $2.2 \pm 2.6$ & .79 \\
Tension & $2.9 \pm 3.2$ & .87 & $2.4 \pm 2.1$ & .74 & $2.5 \pm 2.8$ & .67 \\
Depression & $2.3 \pm 3.7$ & .87 & $1.4 \pm 2.1$ & .18 & $0.9 \pm 2.0$ & .19 \\
Vigour & $9.4 \pm 3.3$ & .62 & $8.6 \pm 4.4$ & .88 & $9.4 \pm 3.1$ & .61 \\
Fatigue & $5.9 \pm 3.5$ & .89 & $6.1 \pm 3.5$ & $<.05$ & $3.1 \pm 2.0$ & $<.05$ \\
Confusion & $3.2 \pm 3.7$ & .75 & $2.9 \pm 2.5$ & .41 & $1.7 \pm 1.7$ & .13 \\
Happiness & $5.0 \pm 2.0$ & .96 & $5.0 \pm 1.9$ & .60 & $4.8 \pm 1.3$ & .65 \\
Calmness & $8.6 \pm 3.4$ & 1.00 & $8.5 \pm 3.0$ & .43 & $9.3 \pm 2.7$ & .40 \\
\hline
\end{tabular}

Note. LW - lightweight category; MW - middleweight category; HW - heavyweight category; $\chi$ - arithmetic mean; SD - standard deviation; $p$-statistically significant difference.

Table 5. Relations (Spearman's rank correlation coefficient) between mood states (BRUMS) and body mass reduction (BM reduction), percentage of body mass reduction (\%BM reduction) and assessment of hydration status (USG refractometer)

\begin{tabular}{|c|c|c|c|c|c|c|c|c|c|}
\hline & \multicolumn{3}{|c|}{ BM reduction } & \multicolumn{3}{|c|}{$\% \mathrm{BM}$ reduction } & \multicolumn{3}{|c|}{ USG refractometer } \\
\hline & LW & MW & HW & LW & MW & HW & LW & MW & $\mathrm{HW}$ \\
\hline Anger & $.55^{*}$ & $.49^{*}$ & -.01 & $.51^{*}$ & $.49^{*}$ & -0.01 & .19 & .28 & -.18 \\
\hline Tension & -.04 & .22 & -.06 & -.05 & .23 & -0.06 & $-.47^{*}$ & .05 & -.43 \\
\hline Depression & .22 & $.50^{*}$ & -.02 & .18 & $.51^{*}$ & -0.02 & -.17 & .16 & -.36 \\
\hline Vigour & .17 & -.16 & .38 & .21 & -.14 & 0.38 & .26 & -.09 & -.20 \\
\hline Fatigue & .17 & $.37^{*}$ & .32 & .14 & $.36^{*}$ & 0.32 & -.40 & $.37^{*}$ & .14 \\
\hline Confusion & -.15 & .26 & .21 & -.18 & .27 & 0.21 & $-.65^{*}$ & .22 & -.01 \\
\hline Happiness & .09 & .11 & .38 & .14 & .13 & 0.38 & $.60^{*}$ & .04 & .15 \\
\hline Calmness & -.18 & $-.36^{*}$ & 017 & -.19 & $-.34^{*}$ & 0.17 & .01 & -.24 & .27 \\
\hline
\end{tabular}

* Correlations are significant at $p<.05$

associated with body mass reduction by the groups of wrestlers.

There were significant differences in body height, body mass and body mass index - BMI (Table 2), which had been expected since the subjects were classified by their weight categories; yet, there was also a significant difference in competition rankings. Further, a significant difference in the reduced body mass was obtained, but that difference lost its significance from the aspect of the reduced body mass percentage.

Table 3 reveals that the applied questionnaire met the reliability criterion across the three groups.

Table 4 makes it obvious that the HW group had significantly higher sense of fatigue then the LW and MW groups.

From the findings presented in Table 5 it is obvious that as for the LW group, BM reduction and $\% \mathrm{BM}$ reduction were positively correlated with anger; USG was positively correlated with happiness and negatively correlated with tension and confusion. For the MW group, BM reduction and $\% \mathrm{BM}$ reduction were positively correlated with anger, depression and fatigue and negatively correlated with calmness, whereas USG was positively correlated with fatigue. As for the HW group, no correlation was found.

\section{Discussion and conclusions}

The groups of wrestlers in our research differed significantly by the expected differences in their anthropological dimensions, but also by the amount of reduced $\mathrm{BM}$ and their ranking in the competition. The wrestlers of the HW group reduced their BM to the smallest extent. The finding is in compliance with previous studies that highlighted that LW and MW wrestlers were the risk groups as regarded RWL (Zubac, Karnincic, \& Zaja, 2015). Also, the HW-group wrestlers achieved signifi- 
cantly better competition ranking than their peers from the LW and MW groups. However, that was most probably due to fewer contestants in the heavier weight categories and not to quality difference. The phenomenon is quite common in wrestling - the fewest contestants appear in the lightest and heaviest weight categories (Baic, Karnincic, \& Sprem, 2015). Out of the 77 investigated subjects, 54 reduced their body mass, and 48 made their weight in the last, pre-competition week. Also, USG values suggest that all the subjects were in the state of dehydration ranging from a mild to a high one (dehydration=USG $>1.020$; Pettersson, et al., 2013). Thus, we could assume that the wrestlers made their body weight by subjecting to RWL. The wrestlers who are in the period of puberty, that is, in the stage of intensive growth and development, should avoid RWL for their health's sake. Unfortunately, inappropriate body mass reduction is a very frequent phenomenon among young wrestlers (Alderman, Landers, Carlson, \& Scott, 2004; Kordi, et al., 2011), and in other combat sports athletes as well (Artioli, et al., 2010; Hall \& Lane, 2001; Jetton, et al., 2013). The current study's findings are in line with the findings of research studies on other combat sports. In the official pre-competition weigh-in young contestants are in a state of dehydration ranging from a mild (USG $\geq 1.020$ ) to a very high dehydration level (USG $\geq 1.030$ ) (Pettersson, et al., 2013). National Collegiate Athletic Association (NCAA) has introduced the Wrestling Weight Certification Program in 1997 after three death incidences (Remick, et al., 1998) as a method to prevent RWL. Research that followed confirmed that legal regulations and prescription of the allowed weight category can mitigate incidence rates of RWL among wrestlers (Artioli, et al., 2010; Davis, et al., 2002). Even in 1990 one paper warned the professional public that dangerous RWL tradition had not changed at all (Steen \& Brownell, 1990). RWL habits have not changed, not even under the influence of a vast body of scientific evidence. Quite the opposite, more drastic examples are evidenced. Mixed martial art (MMA) combatants lose 4.4\% of their BM over 22 hours prior to the weigh-in (Jetton, et al., 2013). If we do not want the 1997 death incidents to re-occur, we should urgently introduce legal regulation of the issue (as already done by NCAA) in all combat sports at all competition levels.

Non-invasive, field methods of hydration assessment and control are very practical in wrestling as well. Our research demonstrated low reliability of urine reagent strips in this sample. Further, the urine colour test was reliable enough if applied to the wrestlers who cut more BM, as was the case in our study with the LW and MW group wrestlers. Namely, if a small amount of BM is reduced, then there is no need to control the hydration status of a wrestler because no high rates of dehydration are expected.

The HW-group wrestlers reduced significantly less BM, therefore they scored expectedly lower on the fatigue scale than the LW- and MW-group wrestlers. Fatigue was the only intergroup distinguishing mood state. It is interesting to underline that fatigue is the most common consequence of dehydration (Benton \& Young, 2015; Moyen, et al., 2015) and that the HW-group wrestlers were hypohydrated as the others (USG refractometer HW=1.027; LW and $\mathrm{MW}=1.026$ ). Therefore, a question arises: why was the HW-group wrestlers' hydration status similar to the status of others although they had cut significantly less BM? It is our assumption that the HW-group wrestlers started their BM cutting later than other wrestlers (since their BM reduction rate was the smallest) and that they used the method of reducing bodily fluids.

When inspecting the relations between RWLinduced dehydration and mood states, common practice is to relate BM loss and mood states. However, BM losses are frequently self-reported, thus consequently subjective. Further, they are strongly affected by seasonal and annual BM sways that can be considerable (Utter, 2001; Lingor \& Olson, 2010). Since wrestlers most frequently reduce their BM on the account of bodily fluids, and the dehydration of about $2 \%$ may induce mood changes (Benton \& Young, 2015; Moyen, et al., 2015), it was expected that a more reliable findings on RWL-induced effects on mood changes would be obtained by the objective method of USG assessment than by the self-reported body mass reduction. However, in our study a transparent picture of the RWL influence on mood states was obtained by means of the BM reduction and \%BM reduction variables, whereas the variable USG yielded a somewhat confusing results in the MW-group wrestlers. The MW-group wrestlers were hypohydrated as the others, but their hypohydration was correlated mostly with positive changes in their mood states. The subjects filled in the mood state questionnaire after the official weigh-in and they were allowed to consume fluids while dealing with the questions. Most negative effects of rapid weight loss disappear only after a 12-hour recovery period (Cengiz, 2015), therefore no recovery could have been reached in such a short period of time. Beverage consumption has effects on mood states (León-Muñoz, et al., 2015), but all the investigated groups of wrestlers consumed water and yet fuzzy relations between mood states and hydration status occurred only in the MW group. Negative mood states are to be expected in relation to painstaking work-outs combined with the restricted food and fluid intake. These findings lack apparent logic; however, they actually corroborate the theory that RWL brings mental advantage to wrestlers (Pettersson, et al., 
2013). Wrestlers, who have been working out painstakingly during the last week in combination with reduction diets and dehydration, do not need to fast any more after the weigh-in and they can actively hydrate after they have reached their previously set goal, so they have all reasons to feel great. However, since ambiguous results were obtained for only one group in only one variable (USG refractometer), we assume that the complexity of bodily fluids regulatory mechanism was responsible for the fuzzy findings, although many other factors could have affected the pre-competition mood states.

Previous research has well evidenced the association between mood states and injury rates (Brewer, 1998); specifically, anger, confusion, fatigue, tension and depression were significantly related to orthopaedic incidents (Galambos, Terry, Moyle, \& Locke, 2005). The wrestlers from MW group achieved significantly higher values in three out of five mood states. Therefore, it is viable to assume that they pertain to a high-risk group as regards potential injuries. Earlier studies also indicated the relation between negative mood and RWL. In boxers, RWL caused increments in anger, fatigue and tension (Hall \& Lane, 2001); in judoists, elevated values of confusion and tension were found (Koral \& Dosseville, 2009); and in wrestlers, increased scores in confusion were obtained (Marttinen, et al., 2011). RWL causes serious health-related issues, especially when its methods are applied to children at the culmination of their growth and development.
Numerous studies, even death accidents, failed to transform harmful habits of RWL. Pre-competition mood states are among aspects being impaired by RWL and a desirable mood state plays an important role in flow during competition in combat sports (Montero-Carretero, Gonzalez-Cutre, MorenoMurcia, Carratala, \& Cervello, 2015).

The aims of the study were to delineate habits of adolescent wrestlers associated with body mass reduction and to establish differences in mood states among wrestlers pertaining to different weight groups. It was established that the investigated adolescent wrestlers resorted habitually to RWL, as reported previously in many research studies cited earlier in the text, despite numerous explorations that had indicated harmful effects of rapid weight cutting.

Wrestlers who reduced their BM significantly scored higher on the sense of fatigue scale. The variables $B M$ reduction and \%BM reduction were in correlation with negative mood states, whereas the hydration status in the group of MW wrestlers was correlated with elevated positive mood states. Therefore, the variables $B M$ reduction and $\% B M$ reduction are recommended to be related with mood states, whereas hydration status should be monitored in young wrestler for their health's sake. Further research should focus on probable consequences of consistent elevation of negative mood states to which adolescent wrestlers are susceptible due to RWL.

\section{References}

Abbey, B.M., Heelan, K.A., Brown, G.A., \& Bartee, R.T. (2014). Validity of hydratrend reagent strips for the assessment of hydration status. Journal of Strength and Conditioning Research, 28(9), 2634-2639.

Alderman, B.L., Landers, D.M., Carlson, J., \& Scott, J.R. (2004). Factors related to rapid weight loss practices among international-style wrestlers. Medicine and Science in Sports and Exercise, 36(2), 249-252.

Armstrong, L.E. (2005). Hydration assessment techniques. Nutrition Reviews, 63(6), 40-54.

Armstrong, L.E. (2007). Assessing hydration status: The elusive gold standard. Journal of the American College of Nutrition, 26(5), 575-584.

Artioli, G.G., Gualano, B., Franchini, E., Scagliusi, F.B., Takesian, M., Fuchs, M., \& Lancha, A.H., Jr. (2010). Prevalence, magnitude, and methods of rapid weight loss among judo competitors. Medicine and Science in Sports and Exercise, 42(3), 436-442.

Artioli, G.G., Franchini, E., Nicastro, H., Sterkowicz, S., Solis, M.Y., \& Lancha, A.H., Jr. (2010). The need of a weight management control program in judo: A proposal based on the successful case of wrestling. Journal of the International Society of Sports Nutrition, 7, 15-15.

Baic, M., Karnincic, H., \& Sprem, D. (2014). Beginning age, wrestling experience and wrestling peak performanceTrends in period 2002-2012. Kinesiology, 46, 94-100.

Benton, D., \& Young, H.A. (2015). Do small differences in hydration status affect mood and mental performance? Nutrition Reviews, 73, 83-96.

Brewer, B.W. (1998). Introduction to the special issue: Theoretical, empirical, and applied issues in the psychology of sport injury. Journal of Applied Sport Psychology, 10(1), 1-4.

Cengiz, A. (2015). Effects of self-selected dehydration and meaningful rehydration on anaerobic power and heart rate recovery of elite wrestlers. Journal of Physical Therapy Science, 27(5), 1441-1444.

Choma, C.W., Sforzo, G.A., \& Keller, B.A. (1998). Impact of rapid weight loss on cognitive function in collegiate wrestlers. Medicine and Science in Sports and Exercise, 30(5), 746-749. 
Davis, S.E., Dwyer, G.B., Reed, K., Bopp, C., Stosic, J., \& Shepanski, M. (2002). Preliminary investigation: The impact of the NCAA Wrestling Weight Certification Program on weight cutting. Journal of Strength and Conditioning Research, 16(2), 305-307.

Degoutte, F., Jouanel, P., Begue, R.J., Colombier, M., Lac, G., Pequignot, J.M., \& Filaire, E. (2006). Food restriction, performance, biochemical, psychological, and endocrine changes in judo athletes. International Journal of Sports Medicine, 27(1), 9-18.

Fernandez-Elias, V.E., Martinez-Abellan, A., Lopez-Gullon, J.M., Moran-Navarro, R., Pallares, J.G., De la CruzSanchez, E., \& Mora-Rodriguez, R. (2014). Validity of hydration non-invasive indices during the weightcutting and official weigh-in for Olympic combat sports. Plos One, 9(4).

Franchini, E., Brito, C.J., \& Artioli, G.G. (2012). Weight loss in combat sports: Physiological, psychological and performance effects. Journal of the International Society of Sports Nutrition, 9.

Galambos, S.A., Terry, P.C., Moyle, G.M., \& Locke, S.A. (2005). Psychological predictors of injury among elite athletes. British Journal of Sports Medicine, 39(6), 351-354.

Hall, C.J., \& Lane, A.M. (2001). Effects of rapid weight loss on mood and performance among amateur boxers. British Journal of Sports Medicine, 35(6), 390-395.

Horswill, C.A., Park, S.H., \& Roemmich, J.N. (1990). Changes in the protein nutritional-status of adolescent wrestlers. Medicine and Science in Sports and Exercise, 22(5), 599-604.

Horswill, C.A., Scott, J.R., Dick, R.W., \& Hayes, J. (1994). Influence of rapid weight-gain after the weigh-in on success in collegiate wrestlers. Medicine and Science in Sports and Exercise, 26(10), 1290-1294.

Jetton, A.M., Lawrence, M.M., Meucci, M, Haines, T.L., Collier, S.R., Morris, D.M., \& Utter, A.C. (2013). Dehydration and acute weight gain in mixed martial arts fighters before competition. Journal of Strength and Conditioning Research, 27(5), 1322-1326.

Karninčić, H., Krstulović, S., \& Baić, M. (2013). The influence of body weight on chosen physiological parameters in wrestling. Journal of Human Kinetics, 37(1), 119-127.

Khanna, G.L., \& Manna, I. (2006). Study of physiological profile of Indian boxers. Journal of Sports Science and Medicine, 5, 90-98.

Klinzing, J.E., \& Karpowicz, W. (1986). The effects of rapid weight-loss and rehydratation on a wrestling performancetest. Journal of Sports Medicine and Physical Fitness, 26(2), 149-156.

Koral, J., \& Dosseville, F. (2009). Combination of gradual and rapid weight loss: Effects on physical performance and psychological state of elite judo athletes. Journal of Sports Sciences, 27(2), 115-120.

Kordi, R., Ziaee, V., Rostami, M., \& Wallace, W.A. (2011). Patterns of weight loss and supplement consumption of male wrestlers in Tehran. Sports Medicine, Arthroscopy, Rehabilitation, Therapy \& Technology: SMARTT, $3(1), 4$.

Lan, M.F., Lane, A.M., Roy, J., \& Hanin, N.A. (2012). Validity of the Brunel Mood Scale for use with Malaysian athletes. Journal of Sports Science and Medicine, 11(1), 131-135.

León-Muñoz, L.M., García-Esquinas, E., López-García, E., Banegas, J.R., \& Rodríguez-Artalejo, F. (2015). Major dietary patterns and risk of frailty in older adults: A prospective cohort study. BioMed Central Medicine, 13, 11. doi: 10.1186/s12916-014-0255-6

Lingor, R.J., \& Olson, A. (2010). Fluid and diet patterns associated with weight cycling and changes in body composition assessed by continuous monitoring throughout a college wrestling season. Journal of Strength and Conditioning Research, 24(7), 1763-1772.

Marttinen, R.H.J., Judelson, D.A., Wiersma, L.D., \& Coburn, J.W. (2011). Effects of self-selected mass loss on performance and mood in collegiate wrestlers. Journal of Strength and Conditioning Research, 25(4), 1010-1015.

McMurray, R.G., Proctor, C.R., \& Wilson, W.L. (1991). Effect of caloric deficit and dietary manipulation on aerobic and anaerobic exercise. International Journal of Sports Medicine, 12(2), 167-172.

Montero-Carretero, C., Gonzalez-Cutre, D., Moreno-Murcia, J.A., Carratala, V., \& Cervello, E.M. (2015). Motivation, mood state, and flow in elite judokas. Revista Mexicana De Psicologia, 32(2), 101-112.

Moyen, N.E.,Ganio, M.S., Wiersma, L.D., Kavouras, S.A., Gray, M., McDermott, B.P., Adams, J.D., Binns, A.P., Judelson, D.A., McKenzie, A.L., Johnson, E.C., Muñoz, C.X., Kunces, L.J., \& Armstrong, L.E. (2015). Hydration status affects mood state and pain sensation during ultra-endurance cycling. Journal of Sports Science, 33(18), 1962-1969.

Oppliger, R.A., Landry, G.L., Foster, S.W., \& Lambrecht, A.C. (1993). Bulimic behaviors among interscholastic wrestlers-A statewide survey. Pediatrics, 91(4), 826-831.

Oppliger, R.A., Magnes, S.A., Popowski, L.A., \& Gisolfi, C.V. (2005). Accuracy of urine specific gravity and osmolality as indicators of hydration status. International Journal of Sport Nutrition and Exercise Metabolism, 15(3), 236-251.

Pettersson, S., Ekstrom, M.P., \& Berg, C.M. (2013). Practices of weight regulation among elite athletes in combat sports: A matter of mental advantage? Journal of Athletic Training, 48(1), 99-108.

Remick, D., Chancellor, K., Pederson, J., Zambraski, E.J., Sawka, M.N., \& Wenger, C.B. (1998). Hyperthermia and dehydration-related deaths associated with intentional rapid weight loss in three collegiate wrestlers-North Carolina, Wisconsin, and Michigan, November-December 1997 (Reprinted from MMWR, Vol. 47, pg. 105-108, 1998). JAMA-Journal of the American Medical Association, 279(11), 824-825. 
Saarni, S.E., Rissanen, A., Sarna, S., Koskenvuo, M., \& Kaprio, J. (2006). Weight cycling of athletes and subsequent weight gain in middleage. International Journal of Obesity, 30(11), 1639-1644.

Shirreffs, S.M. (2003). Markers of hydration status. European Journal of Clinical Nutrition, 57, S6-S9.

Smith, N.J. (1984). Weight control in the athlete. Clinics in Sports Medicine, 3(3), 693-704.

Song, T.M., \& Garvie, G.T. (1980). Anthropometric, flexibility, strength, and physiological measures of Canadian wrestlers and comparison of Canadian and Japanese Olympic wrestlers. Canadian Journal of Applied Sport Sciences. Journal Canadien des Sciences Appliquees au Sport, 5(1), 1-8.

Steen, S.N., \& Brownell, K.D. (1990). Patterns of weight loss and regain in wrestlers: Has the tradition changed? Medicine and Science in Sports and Exercise, 22(6), 762-768.

Stevens, M.J., Lane, A.M., \& Terry, P.C. (2006). Mood profiling during Olympic qualifying judo competition: A case study testing transactional relationships. Journal of Sports Science and Medicine, 5, 143-151.

Tipton, C.M., \& Tcheng, T.K. (1970). Iowa Wrestling Study-Weight loss in high school students. JAMA-Journal of the American Medical Association, 214(7), 1269-1274.

Utter, A.C. (2001). The New National Collegiate Athletic Association Wrestling Weight Certification Program and sport-seasonal changes in body composition of college wrestlers. Journal of Strength and Conditioning Research, 15(3), 296-301.

Weber, A.F., Mihalik, J.P., Register-Mihalik, J.K., Mays, S., Prentice, W.E., \& Guskiewicz, K.M. (2013). Dehydration and performance on clinical concussion measures in collegiate wrestlers. Journal of Athletic Training, 48(2), 153-160.

Yoon, J. (2002). Physiological profiles of elite senior wrestlers. Sports Medicine, 32(4), 225-233.

Zhang, C.Q., Si, G.Y., Chung, P.K., Du, M.M., \& Terry, P.C. (2014). Psychometric properties of the Brunel Mood Scale in Chinese adolescents and adults. Journal of Sports Sciences, 32(15), 1465-1476.

Zubac, D., Karninčić, H., \& Žaja, M. (2015). Hydration status assessment among elite youth amateur boxers. Journal of Sports Medicine and Physical Fitness, (E-pub ahead of print, Feb 2015).

Submitted: October 2, 2015

Accepted: July 18, 2016

Correspondence to:

Prof. Hrvoje Karninčić, Ph.D.

Faculty of Kinesiology, University of Split

Teslina 6, 21000 Split, Croatia

Phone: 385915560730

E-mail: hrvojek@kifst.hr 